Rechtsmedizin $2022 \cdot 32: 241-248$ https://doi.org/10.1007/s00194-021-00543-z Angenommen: 13. Oktober 2021 Online publiziert: 3. Dezember 2021 () Der/die Autor(en) 2021

\section{Dekonvolution von Mischspuren nach vollständig kontinuierlichem Modell}

\author{
Beate Dika · Jan Dreßler · Jeanett Edelmann · Michael Kohl \\ Institut für Rechtsmedizin, Universität Leipzig, Leipzig, Deutschland
}

\title{
Zusammenfassung
}

Hintergrund: Bei der Untersuchung von Mischspuren können stochastische Effekte die Beurteilung einer Spurenlegerschaft beeinträchtigen. Daher finden immer mehr softwarebasierte Lösungen Einzug in die Spurenuntersuchung, die durch Berücksichtigung biologischer Parameter eine Hilfestellung bei der Ableitung von Einzelprofilen bieten sollen. Im Rahmen der Studie wurde eine wissenschaftliche Validierung der Mischspurenanalyse-Software Genoproof ${ }^{\circledR}$ Mixture 4 (GPM4, Qualitype $\mathrm{GmbH}$, Dresden, Deutschland) durchgeführt.

Material und Methoden: Die in unterschiedlichen Mischungsverhältnissen vorliegenden 2- und 3-Personen-Mischspuren wurden künstlich unter Verwendung isolierter $\mathrm{CD}^{+}{ }^{+}$-Lymphozyten von 9 Spendenden erzeugt. Nach Erstellung der STRProfile wurden die Mischspuren mittels GPM4 im Hinblick auf die Dekonvolution ausgewertet.

Ergebnisse: In den 2-Personen-Mischspuren mit klarer Unterscheidung von Hauptund Nebenkomponente wurde von der Software in der Großzahl der untersuchten STRSysteme die richtige Genotypkonstellation (GTK) der Komponenten abgeleitet, oftmals mit einer Wichtung $>90 \%$. In den anteilsähnlicheren Mischspuren wurden zunehmend nichtzutreffende Allelableitungen beobachtet. Eine Abnahme der Performance in Bezug auf die Ableitung der richtigen GTK zeigten die 3-Personen-Mischspuren. Faktoren wie Mischkomposition und Homo- und Heterozygotie in den genetischen Profilen hatten nachweislich einen Einfluss auf die Auftrennung der Mischspuren. Diskussion: Mischspuren, die keine klare Unterscheidung von Haupt- und Nebenkomponente erlauben, stellen eine Schwierigkeit bei der Dekonvolution dar. In diesen Fällen ist eine Differenzierung der Peakhöhen detektierter Allele nur schwer möglich, da diese bei Anteilsgleichheit beider Komponenten eine komparable Intensität aufweisen. Ein deutlicher DNA-Mengen-Unterschied der Komponenten ist für die Berechnung von Vorteil.

\section{Schlüsselwörter}

Forensische Molekulargenetik - Mischspurenanalyse - Dekonvolution · Probabilistisches Rechenmodell · Genoproof Mixture

In der Forensik kommt der Mischspurenanalyse eine große Bedeutung zu, da aufgrund sensitiver Untersuchungsmethoden auch kleinste DNA-Mengen analysiert werden können. Gerade bei Hautkontaktspuren treten häufig DNAMischungen mehrerer Personen auf. Dies stellt eine Herausforderung für die Ableitung genetischer Einzelprofile dar. Die Auswertung und Interpretation von Mischspuren kann durch die Verwendung spezieller Software unterstützt werden. Sie leistet Hilfestellung bei der Ableitung von Merkmalsprofilen beteiligter Personen und kann zur Berechnung der Verursacherwahrscheinlichkeit herangezogen werden.

\section{Einleitung}

Spuren, die mehr als 2 Allele in mindestens 2 DNA-Systemen zeigen, werden als Mischspur bezeichnet [9]. Diese entstehen v. a. durch Vermischung kernhaltigen Zell- 
Tab. 1 Mischverhältnisse für 2-und 3-Personen-Mischungen (in Prozent)

\begin{tabular}{|c|c|c|c|c|c|c|}
\hline \multicolumn{3}{|c|}{ 2-Personen-Mischungen } & \multicolumn{4}{|c|}{ 3-Personen-Mischungen } \\
\hline- & $\begin{array}{l}\text { Anteil } \\
\text { Person } 1\end{array}$ & $\begin{array}{l}\text { Anteil } \\
\text { Person 2 }\end{array}$ & - & $\begin{array}{l}\text { Anteil } \\
\text { Person } 1\end{array}$ & $\begin{array}{l}\text { Anteil } \\
\text { Person } 2\end{array}$ & $\begin{array}{l}\text { Anteil } \\
\text { Person } 3\end{array}$ \\
\hline 1 & 80 & 20 & 1 & 50 & 30 & 20 \\
\hline 2 & 75 & 25 & 2 & 50 & 20 & 30 \\
\hline 3 & 70 & 30 & 3 & 30 & 50 & 20 \\
\hline 4 & 65 & 35 & 4 & 20 & 50 & 30 \\
\hline 5 & 60 & 40 & 5 & 30 & 20 & 50 \\
\hline 6 & 55 & 45 & 6 & 20 & 30 & 50 \\
\hline 7 & 45 & 55 & \multicolumn{4}{|l|}{-} \\
\hline 8 & 40 & 60 & \multicolumn{4}{|l|}{-} \\
\hline 9 & 35 & 65 & \multicolumn{4}{|l|}{-} \\
\hline 10 & 30 & 70 & \multicolumn{4}{|l|}{-} \\
\hline 11 & 25 & 75 & \multicolumn{4}{|l|}{-} \\
\hline 12 & 20 & 80 & \multicolumn{4}{|l|}{-} \\
\hline
\end{tabular}

materials wie z.B. Schleimhaut- und Spermienzellen nach einem sexuellen Missbrauch. Der Nachweis von max. 4 Allelen/ System lässt im Elektropherogramm (EPG) auf mindestens 2 unterschiedliche Spurengebende schließen, beim Nachweis von max. 6 Allelen sind es mindestens 3 unterschiedliche Spurengebende. Die Bewertung von Mischprofilen im Hinblick auf die Ableitung von Einzelprofilen ist Gegenstand forensischer Untersuchungen. Dabei kann die Profilinterpretation mithilfe des EPG durch das Auftreten technisch und/oder biologisch bedingter Artefakte, die während der Amplifikation der STRMarker entstehen, erschwert werden. Dazu gehören z. B. "Stutter"-Effekte und „Dropout"-Ereignisse $[1,6,13]$, die dazu führen, dass nicht alle einer Person eindeutig zuordenbaren Merkmale identifiziert werden und diese somit als Spurenlegende ausgeschlossen werden kann. In einer Studie von Dror und Hampikian konnte gezeigt werden, dass die Interpretation von DNA-Mischungen durch Sachverständige ein gewisses Maß an Subjektivität mit sich bringt und von individuellen Unterschieden wie Erfahrung, Persönlichkeit und Motivation abhängig sein kann [2]. Aus diesem Grund finden immer mehr softwarebasierte Lösungen Einzug in die Routine der Spurenuntersuchung, wodurch Objektivität gewährleistet wird und eine Berücksichtigung biologischer Parameter bei der Auswertung Unterstützung bieten soll.

\section{Genoproof ${ }^{\circledR}$ Mixture 4}

Genoproof ${ }^{\circledR}$ Mixture 4 (GPM4) der Fa. Qualitype $\mathrm{GmbH}$ (Dresden, Deutschland) ist eine Software zur Auswertung von STRAnalysen komplexer Mischspuren. Dabei stehen probabilistische und nichtprobabilistische Rechenmodelle zur Verfügung, die für die Interpretation von Mischspuren eingesetzt werden können [4, 7]. Die Software GPM4 bietet die Möglichkeit zur Dekonvolution von Mischspuren, bei der die genetischen Profile der an der Mischung beteiligten Personen rechnerisch aufgetrennt und unter Angabe der wahrscheinlichsten Genotypkonstellationen (GTK) ermittelt werden. Die softwarebasierte Dekonvolution kann dafür genutzt werden, subjektiv durch Begutachtende aus Mischspurenmustern abgeleitete Merkmalsprofile sowie darüber hinausgehende Ableitungen von Merkmalsprofilen aus komplexen Mischmustern ohne oder mit Einbeziehen von Vergleichspersonen zu bestätigen.

Im Rahmen dieser Studie wurde eine wissenschaftliche Validierung von Genoproof ${ }^{\circledR}$ Mixture 4 unter Verwendung künstlich hergestellter 2- und 3-PersonenMischungen durchgeführt. Die Ergebnisse wurden im Hinblick auf die Dekonvolution ausgewertet, wobei die Grenzen und Möglichkeiten der Software in Bezug auf die korrekte Zuweisung der GTK der Spurenverursachenden analysiert wurden. Aus den Erkenntnissen der Studie soll die Anwendbarkeit von GPM4 als unterstüt- zendes Analyse-Tool in der Auswertung von Mischspuren hinsichtlich einer potenziellen Nutzung durch Sachverständige in realen Spurenfällen beurteilt werden.

\section{Methodik}

\section{Probenmaterial}

Es wurden etwa $15 \mathrm{ml}$ Vollblut von insgesamt 9 freiwilligen Probanden und Probandinnen (Bezeichnungen $A$ bis I) mit bekanntem genetischen Profil entnommen. In Kooperation mit dem Institut für Transfusionsmedizin (Universitätsklinikum Leipzig AöR) wurden kernhaltige Bestandteile des Bluts (CD4+-Lymphozyten) isoliert und für die Erstellung definierter Zellmischungen eingesetzt.

Für die Entnahme und Verwendung des Probenmaterials wurde vor Beginn der Studie ein positives Votum der Ethikkommission der Medizinischen Fakultät der Universität Leipzig (Nummer 45320-ek) eingeholt. Die Teilnehmenden wurden über den Ablauf der Studie informiert, eine schriftliche Einverständniserklärung zur genetischen Untersuchung liegt vor.

\section{Erstellung der Mischspuren}

Aus den $\mathrm{CD}^{+}$-Zelllösungen der Teilnehmenden wurden anhand der Zellzahlen unterschiedliche 2- und 3-Personen-Mischspuren mit verschiedenen Mischungsverhältnissen erstellt. Für die 2-Personen-Spuren wurden die Zellen der Personen $A B, A C, B C$ sowie $D E, D F$, EF in jeweils 12 Mischungsverhältnissen miteinander kombiniert $(n=72)$. Die 3-PersonenMischungen setzten sich aus den Zellen der Personen ABC, DEF und GHI zusammen und lagen in 6 Mischungsverhältnissen vor $(n=18)$ ( $\bullet$ Tab. 1). Die Mischungsverhältnisse wurden so gewählt, dass jeweils die DNA einer Person als Hauptkomponente in der Mischung vorliegt, die DNA der weiteren Person/Personen als Nebenkomponente. Bei der Zusammenstellung der Mischmuster anhand der bekannten genetischen Profile der Probanden und Probandinnen wurden vorzugsweise Konstellationen gewählt, bei denen sich Mischprofile mit sowohl überwiegend heterozygoten als auch überwiegend homozygoten Allelkombinationen ergaben. 
Tab. 2 Übersicht der Homo-/ Heterozygotie $^{\text {a }}$

\begin{tabular}{|l|l|l|}
\hline $\begin{array}{l}\text { Per- } \\
\text { son }\end{array}$ & $\begin{array}{l}\text { Heterozygote } \\
\text { Allelpaare }\end{array}$ & $\begin{array}{l}\text { Homozygote } \\
\text { Allelpaare }\end{array}$ \\
\hline A & 14 & 2 \\
\hline B & 10 & 6 \\
\hline C & 13 & 3 \\
\hline D & 7 & 9 \\
\hline E & 9 & 7 \\
\hline F & 14 & 2 \\
\hline G & 14 & 2 \\
\hline H & 14 & 2 \\
\hline I & 14 & 2 \\
\hline${ }^{a} 16$ STR-Systeme &
\end{tabular}

\section{STR-Analyse der Mischspuren}

Die Extraktion der in den Mischspuren enthaltenen genomischen DNA erfolgte mit dem Nucleospin ${ }^{\circledR}$ Tissue Kit der Fa. Macherey-Nagel (Düren, Deutschland). In einer qRT-PCR wurde die Konzentration der extrahierten DNA-Proben bestimmt. Hierfür wurde das PowerQuant ${ }^{\circledR}$-System der Fa. Promega (Madison, WI, USA) verwendet. Die simultane Amplifikation der 16 untersuchten STR-Systeme und des geschlechtsbestimmenden Markers Amelogenin wurde in einer Doppelbestimmung mittels Multiplex-PCR unter Verwendung der Kits PowerPlex ${ }^{\circledR}$ ESI 17 Pro System und PowerPlex ${ }^{\circledR}$ ESX 17 Pro System der Fa. Promega durchgeführt. In der PCR wurde eine DNA-Menge von 0,5 ng eingesetzt, um stochastische Effekte bewusst zu vermeiden. Basierend auf Validierungsstudien der verwendeten PCR-Kits $[10,11]$ wurden keine Drop-outs bei einer eingesetzten DNAMenge von $100 \mathrm{pg}$, was dem geringsten Anteil von $20 \%$ einer Komponente in den 2-und 3-Personen-Mischungen entspricht, beobachtet.

Anschließend erfolgte eine Fragmentanalyse mittels Kapillarelektrophorese (3130 Genetic Analyzer, Fa. Applied Biosystems, Waltham, RI, USA), bei der die den Kits (ESI/ESX) entsprechenden Allelleitern mitgeführt wurden. Die Auswertung der Rohdaten aus der Fragmentanalyse der Mischspuren wurde mit der Software GeneMapper $^{\circledR}$ ID-X Version 1.5 (Applied Biosystems) sowie der EasyReadTechnologie von Genoproof ${ }^{\circledR}$ Mixture 4 durchgeführt. Dabei werden bei beiden Softwares unter Einbeziehung der proben- internen Größenstandards Parameter wie Fragmentlänge, Peakhöhe und -fläche der in der Mischspur enthaltenen Allele erhoben und in Form eines EPG dargestellt. Die Detektionsgrenze für die Peakerkennung betrug in allen 4 Farbkanälen 50 RFU. Zudem wurden einige Einstellungen in Bezug auf die Artefakterkennung des „Allele-calling"-Algorithmus in GPM4 abweichend von der Herstellerempfehlung modifiziert, wodurch Artefakte wie Stutter-Peaks als solche im EPG gekennzeichnet wurden und keine Allelzuweisung erhielten.

\section{Dekonvolution}

Die Auftrennung der Genotypen der an der Spur beteiligten Personen sowie die Wichtung der wahrscheinlichsten Genotypkonstellationen wird durch die Modellierung der Peakhöhen der GTK, basierend auf der Berücksichtigung zahlreicher Parameter, erzielt. Dazu gehören die DNA-Menge, die DNA-Degradation, die Fragmentlänge, die Amplifikationseffizenz und das Verhältnis von Homo- und Heterozygotie der an der untersuchten Spur beteiligten Personen. Basierend auf dem Markov-Chain-MonteCarlo-Verfahren [3] werden die Parameter durch einen stochastischen Zufallsprozess geschätzt. Dabei wird die Wahrscheinlichkeit berechnet, dass die erwarteten Peakhöhen möglichst gut mit den beobachteten Peakhöhen für eine zufällige Parameterkombination und eine zufällige GTK übereinstimmen [7]. Ein Wichtungswert von $90 \%$ dient als Orientierungswert für die Wahrscheinlichkeit, dass die berechnete GTK mit der tatsächlichen GTK übereinstimmt. Nach Empfehlung der Spurenkommission (GEDNAP) wurde bei den Berechnungen die europäische Standardpopulation als Referenzpopulation angegeben [12].

In GPM4 wird nach jeder Berechnung eine Konvergenzanalyse bereitgestellt, die Informationen zu den in der Mischspur vorliegenden komponentenbezogenen DNA-Mengen beinhaltet. Diese DNAMengen entsprechen dem von GPM4 angenommenen Mischungsverhältnis dieser Spur. Als Hauptkomponente wird die bei der Konvergenzanalyse mit der höchsten DNA-Menge in der Mischung vorliegende Komponente bezeichnet, die weitere/weiteren Komponente/Komponenten der Mischspur als Nebenkomponente/ Nebenkomponenten.

Die Wichtungen der GTK der 2- und 3-Personen-Mischspuren wurden jeweils 5-mal mittels GPM4 unter Verwendung von 4 Markov-Ketten berechnet und aus den errechneten Wichtungen der Mittelwert gebildet. Des Weiteren wurden die bei jedem Durchlauf ermittelten Genotypen mit den tatsächlichen Genotypen der beteiligten Personen hinsichtlich auftretender Diskrepanzen in den Allelzuweisungen analysiert.

\section{Dekonvolution mit Vergleichs- material}

Beim sog. Vergleichsmaterial (VM) handelt es sich um DNA-Material einer beispielsweise tatverdächtigen Person, das zum Beweis einer Spurenlegerschaft im Rahmen eines Kriminalfalls untersucht wird. Um zu überprüfen, inwiefern der Einsatz von VM einen Einfluss auf die Ermittlung der Einzelprofile der in den Mischspuren beteiligten Personen mittels GPM4 hat, wurden jeweils die genetischen Profile der Komponenten mit $30 \%$-Anteil der 3 Mischspuren A30B20C50, D30E20F50 und $\mathrm{G} 50 \mathrm{H} 20130$ als bekannte Verursachende in die Berechnung der Dekonvolution mit einbezogen. Die Rechnung wurde einmal durchgeführt und die ermittelten GTK mit dem tatsächlichen Personenprofil abgeglichen.

\section{Ergebnisse}

\section{2-Personen-Mischspuren}

Das genetische Profil der Person A besteht überwiegend aus heterozygoten Allelkombinationen, während Person B in 6 STRSystemen eine homozygote Ausprägung aufweist (•Tab. 2). Der jeweilige Probenname beinhaltet die an der Mischung beteiligten Personen und deren Anteil innerhalb der Mischung (Beispiel: A80B20; Person A: $80 \%$, Person B: $20 \%$ ). Die Bewertung der Allelzuweisung in Bezug auf ihre Richtigkeit erfolgte unter Annahme der theoretischen Mischungsverhältnisse, die bei der Erstellung der 2- und 3-Personen-Mischspuren angestrebt wurden (• Tab. 1).

In den $A B-M i s c h s p u r e n$ mit eindeutiger Haupt- und Nebenkomponente (A80B20, A75B25; A20B80, A25B75, A30B70) stimm- 


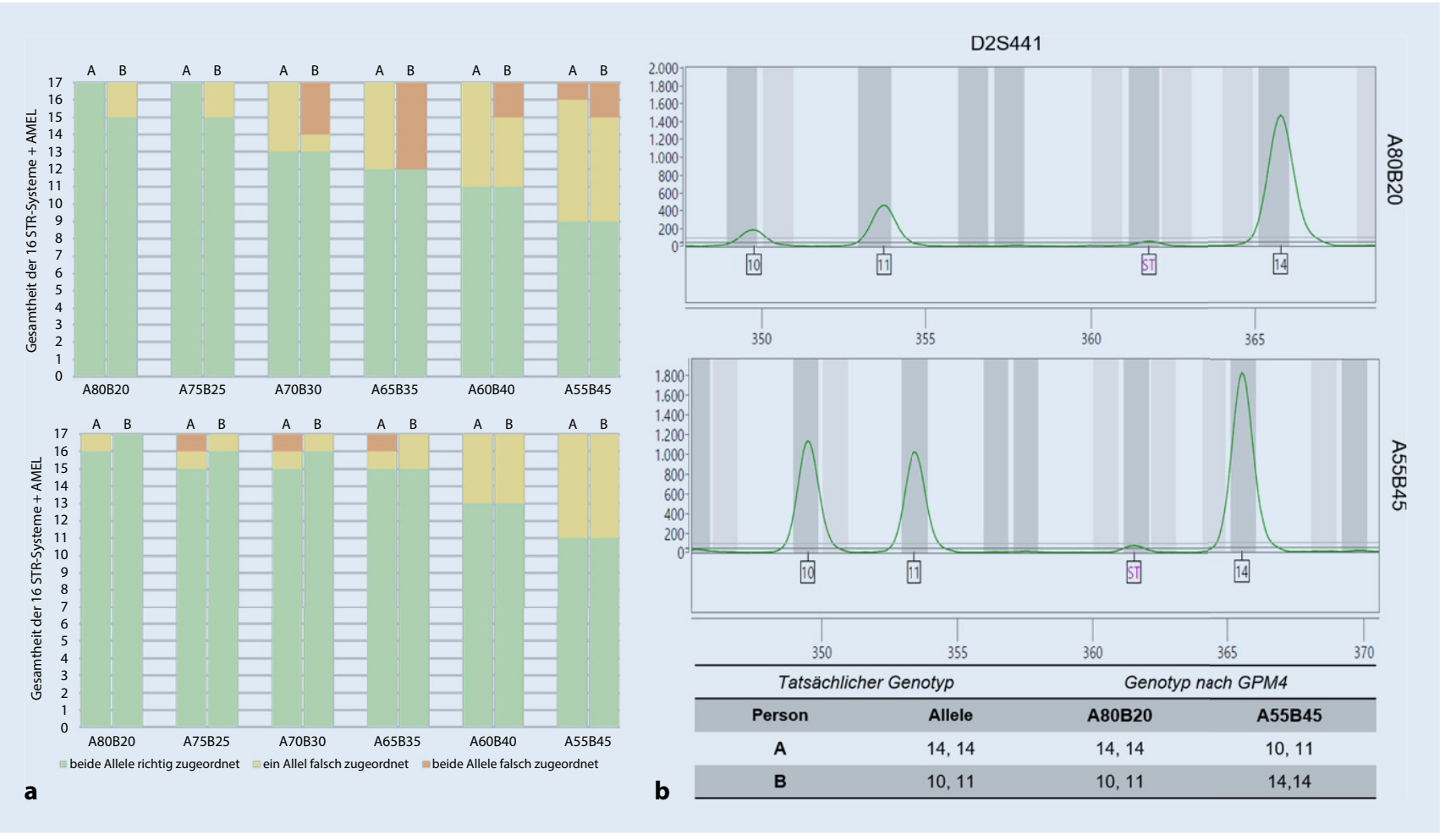

Abb. 1 \ Ableitung der Genotypkonstellationen (GTK) in den Mischspuren mit Personen A (oben) und B (unten) als Hauptkomponente. a Anteile der korrekt/inkorrekt zugeordneten STR-Systeme/Amelogenin; grün = beide Allele korrekt zugeordnet, $g e l b=$ ein Allel korrekt zugeordnet, $r o t=$ beide Allele falsch zugeordnet. b EPG-Ausschnitt des STR-Markers D2S441 der Mischspuren A80B20 und A55B45. Die Allelpeaks 10, 11 und 14zeigen in beiden Mischspuren unterschiedliche Fluoreszenzintensitäten, abhängig vom jeweiligen Anteil der Komponenten A und B. ST "stutter", GPM4 "Genoproof Mixture 4"

ten die von GPM4 ermittelten Genotypen bis auf einzelne Allele mit den tatsächlichen Genotypen der Person A und B überein. Dabei wurden die zugeordneten Genotypen der jeweiligen Hauptkomponente im Großteil der STR-Systeme mit $>90 \%$ gewichtet, während die abgeleiteten GTK der Nebenkomponente öfter eine Wichtung $<90 \%$ in einigen Systemen aufwiesen. Es wurde eine Abnahme der Wichtungswerte hin zu den anteilsähnlicheren Mischungen beobachtet, in denen die GTK bei beiden Personen mit Werten $<90 \%$ gewichtet wurden. Es konnte festgestellt werden, dass eine Wichtung $<90 \%$ nicht zwingend bedeutet, dass die abgeleitete GTK nicht dem tatsächlich vorliegenden Genotyp entspricht. Im Gegenzug kann eine über $>90 \%$ gewichtete Allelableitung dem tatsächlichen Genotyp widersprechen.

In Abb. 1 sind die Ergebnisse der Dekonvolution mittels GPM4 hinsichtlich der richtigen Zuordnung der in den $A B-$ Mischspuren ( $n=12)$ enthaltenen Allele zu den Einzelprofilen der Spurenbeteiligten A und B dargestellt. Es wird die Richtigkeit der Allelzuordnungen in den 16 autosomalen STR-Systemen sowie im gonosomalen System AMEL aufgezeigt. Eine korrekte Zuordnung der Allele (grüne Balken) in allen Systemen wurde für Person $A$ in den Mischungen A80B20 und A75B25 sowie für Person $B$ in der Mischung A20B80 beobachtet. Eine inkorrekte Genotypisierung einiger STR-Systeme, also die Ableitung von einem (gelbe Balken) oder 2 (rote Balken) der Person nicht zugehörigen Allele, war v.a. in den anteilsähnlicheren Mischspuren zu beobachten. Grundlegend konnte festgestellt werden, dass eine inkorrekte Genotypisierung einer Person der Mischung oftmals dazu führt, dass in den gleichen Systemen die Allele der anderen Person ebenfalls nicht mit dem tatsächlichen Genotyp übereinstimmen. Die Bedeutung der Peakhöhendifferenz, basierend auf dem Mischungsverhältnis (80:20 vs. 55:45), bei der Ableitung der GTK für die Beteiligten A und B im Marker D2S441 verdeutlicht $\bullet$ Abb. 1b. Eine geringere Diffe- renz zwischen den Allelpeaks begünstigte eine fehlerhafte Ableitung von Allelen.

Im Zuge der Mischspurenanalyse aufgetretene Stutter-Produkte wurden von der Software anhand der EasyRead-Technologie erfolgreich als solche identifiziert, im EPG entsprechend gekennzeichnet und nicht als Allele gewertet (- Abb. 1b). Somit zeigte das Auftreten von Stutter-Produkten keinen Einfluss auf die Ableitung der wahrscheinlichsten GTK mittels GPM4. Mischspuren, in denen technisch bedingte Drop-out-Ereignisse auftraten, wurden einer wiederholten STR-Analyse unterzogen, sodass der Einfluss von „allelic dropouts" hinsichtlich der Zuweisung der Genotypen im Rahmen dieser Studie nicht weiter untersucht wurde. Durch den Ausfall eines Allels oder mehrerer Allele an den untersuchten Loci ist jedoch mit einer höheren Fehlerquote in Bezug auf die GTKAbleitung der Spurengebenden zu rechnen. 


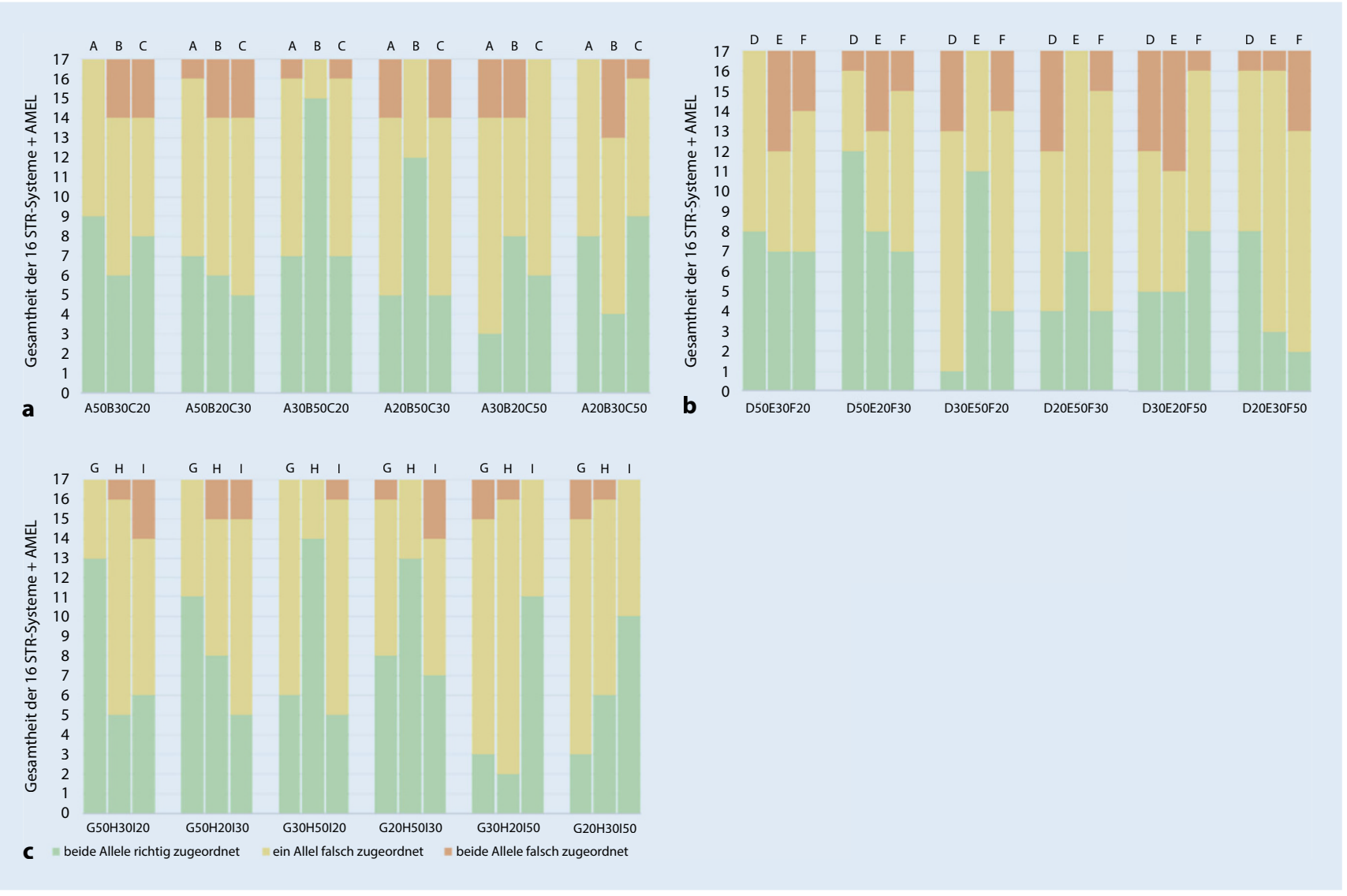

Abb. 2 \ Ableitung der GTK in den 3-Personen-Mischspuren. a Spurenbeteiligung von Personen A, B und C. b Spurenbeteiligung von Personen D, E, F. c Spurenbeteiligung von Personen G, H, I

\section{3-Personen-Mischspuren}

Die Ergebnisse der Dekonvolution von künstlich erzeugten 3-Personen-Mischspuren sind in $\mathbf{0}$ Abb. 2 dargestellt. Dabei wurde bei den unterschiedlichen Personenkonstellationen ( $A B C$, DEF und GHI) in Bezug auf die Performance der GTKAbleitung mittels GPM4 eine Abhängigkeit von Homo-/Heterozygotie sowie der Mischkomposition beobachtet.

Im Vergleich zu den 2-Personen-Mischspuren wiesen die von der Software abgeleiteten GTK für die beteiligten Personen der 3-Personen-Mischspuren meist eine Wichtung $<90 \%$ auf. Vor allem in der Probe A20B30C50 wurden die Genotypen der Nebenkomponenten A und B mit Wahrscheinlichkeiten $<30 \%$ gewichtet. In der Spur D30E50F20 wurden die Allelkombinationen der Hauptkomponente $\mathrm{E}$ in einigen STR-Systemen mit $>90 \%$ gewichtet und kongruierten mit dem tatsächlich vorliegenden Genotyp. Die genetischen Einzelprofile der Nebenkomponenten D und $\mathrm{F}$ dagegen wurden von der Software in fast allen Markern nicht korrekt abgeleitet (- Abb. 2b, D30E50F20). Die von GPM4 zugewiesenen GTK der Hauptkomponente $\mathrm{H}$ aus Spur $\mathrm{G} 30 \mathrm{H} 50120$ wurden in 11 von 17 Systemen mit einem Wert $>90 \%$ gewichtet. Darunter befanden sich 7 GTK, für die ein Wichtungswert von $99 \%$ berechnet wurde und eine Übereinstimmung mit dem tatsächlichen Profil der Person $\mathrm{H}$ in den entsprechenden STR-Systemen vorlag. Die Nebenkomponenten G und I zeigten insgesamt geringere Wichtungen und einige Fehlableitungen von Allelen auf.

Die Anteile der richtig abgeleiteten STRSysteme fielen im Vergleich zu den 2-Personen-Mischspuren deutlich geringer aus. Es ließ sich beobachten, dass die Anteile der STR-Marker mit dem richtigen Genotyp (grüne Balken) in fast allen Mischspuren bei der jeweiligen Hauptkomponente höher waren als bei den Nebenkomponenten, für die des Öfteren die Ableitung 2 nichtzugehöriger Allele beobachtet wurde (rote Balken). Für Hauptkomponenten, die ein genetisches Profil mit einer höheren Zahl an homozygoten Allelpaaren besaßen ( $B$, D), wurde von der Software in vergleichsweise mehr STR-Systemen eine GTK abgeleitet, die dem tatsächlichen Genotyp entsprach. Zu einer höheren Fehleranfälligkeit in Bezug auf die Ableitung von Allelpaaren kam es jedoch beim Vorliegen einer Mischspur mit einem höheren Anteil an homozygoter Allelausprägung (DEF). Die Anzahl der Marker, in denen eine Fehlableitung beider Allele detektiert wurde (rote Balken), war in den GHI-Mischspuren gegenüber den $A B C$ - und DEF-Mischspuren sichtbar geringer (- Abb. 2c).

Das Beispiel in - Abb. 3 verdeutlicht die Herausforderung in Bezug auf die Ableitung der richtigen GTK beim Auftreten überlagernder Allele. Im Marker FGA der Mischspur G30H50I20 kommt das Allel 21 bei allen 3 Spurengebenden vor, wodurch dieser Peak die höchste Signalintensität aufwies und somit der stärksten Komponente 1 (Person H) als homozygote Kombination zugewiesen wurde. Das zu Per- 


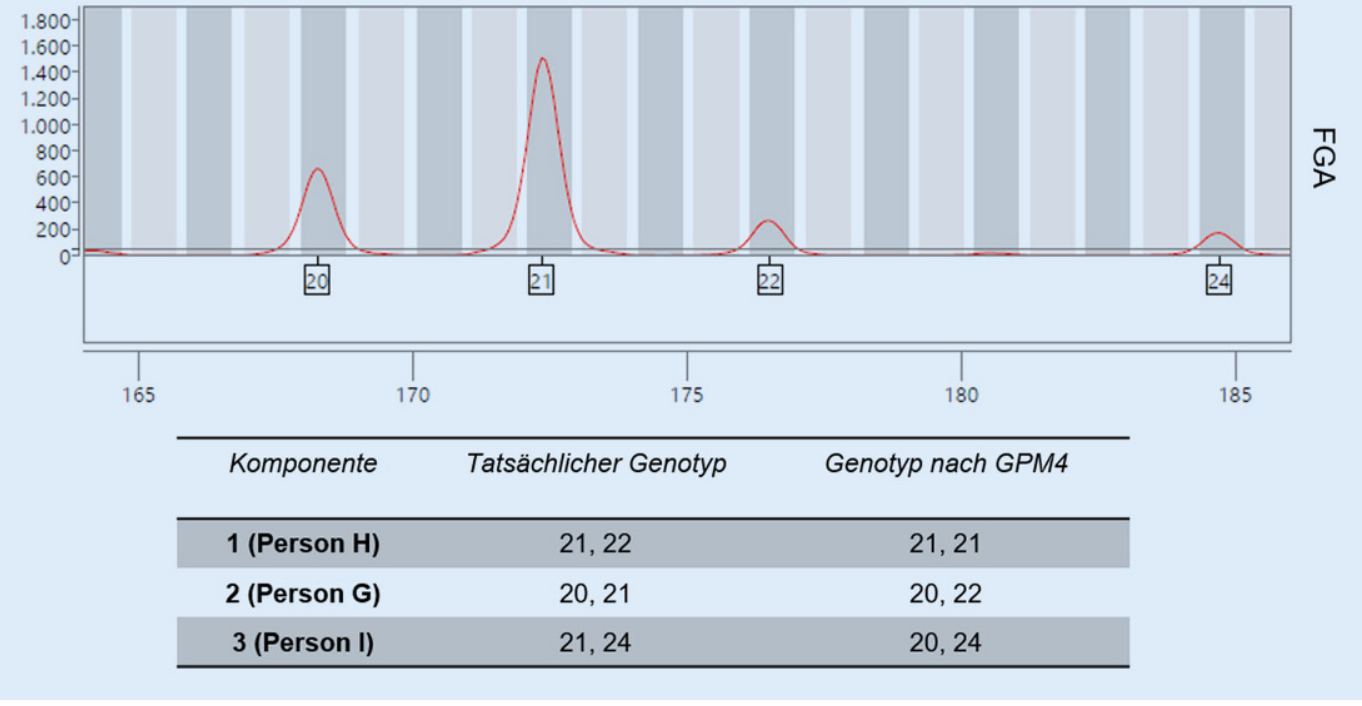

Abb. $3 \triangleleft$ Marker FGA der Mischspur G30H50I20. Fluoreszenzintensität der Allelpeaks 20, 21, 22 und 24 und deren Zuordnung zu den Spurengebenden $\mathrm{G}, \mathrm{H}$ und I son $\mathrm{H}$ gehörige Allel 20 stellte den Peak mit der zweithöchsten Fluoreszenzintensität des Systems dar und wurde durch GPM4 den beiden Nebenkomponenten 2 und 3 (Personen $\mathrm{G}$ und I) zugeschrieben. Von der Software wurde die Zuweisung des Allels 21 zum Genotyp von $\mathrm{H}$ mit einem Wichtungswert von $91,82 \%$ bewertet und entsprach damit innerhalb der GHI-Mischspuren der einzigen abgeleiteten GTK, die mit einer Wichtung $>90 \%$ nicht mit der tatsächlichen GTK übereinstimmte.

\section{Dekonvolution mit Vergleichs- material}

Die zuvor aufgeführten Berechnungen der Dekonvolution erfolgten unter der Annahme von 3 unbekannten Spurenverursachenden, also unter Ausschluss von Vergleichsmaterial. Die genetischen Profile der anteilig am zweithöchsten vorliegenden Komponenten (30\%) A, D und I wurden als VM in der Berechnung mitgeführt, wodurch die GTK dieser Komponenten bereits vorgegeben waren. Die Wichtungen der betreffenden Komponente wurden im Ergebnis mit 100\% wiedergegeben.

In diesem Fall konnte für alle 3 Mischspuren eine steigende Effizienz in Bezug auf die richtige Ableitung der Allelkombinationen verzeichnet werden (• Abb. 4). Des Weiteren zeigte sich ein Anstieg der Wichtungswerte für die abgeleiteten GTK der einzelnen Komponenten. Bei dieser Berechnung konnten für die jeweiligen
Hauptkomponenten (C, F, G) Wichtungen $>90 \%$ für die zugewiesenen Allelpaare beobachtet werden (vorher teilweise $<50 \%$ ).

Die Berücksichtigung von VM zeigte einen deutlichen Kontrast zu den Ergebnissen der Dekonvolution ohne VM. Der Anteil der Systeme mit der Zuweisung eines falschen Allels verringerte sich deutlich.

\section{Diskussion}

Die Interpretation einer Mischspur stellt im Vergleich zu einer Einzelspur eine Herausforderung dar, da durch den Nachweis mehrerer Allele in einem System keine eindeutigen Rückschlüsse auf die Genotypkonstellation eines/einer Spurenverursachenden gezogen werden können. Das Auftreten stochastischer Effekte kann die Ableitung von Einzelprofilen durch Sachverständige dabei zunehmend erschweren. Bislang werden lediglich Berechnungen zur Verursacherwahrscheinlichkeit als gerichtsverwertbares Beweismittel zur Klärung einer Täterschaft zugelassen, wodurch die wissenschaftliche Validierung von Mischspurenanalyse-Softwares immer mehr an Bedeutung gewinnt.

Die Herleitung der wahrscheinlichsten GTK geschieht über den Abgleich der für diese GTK erwarteten Peakhöhen mit den beobachteten Peakhöhen der in der Mischspur detektierten Allele. Die Höhe eines Allelpeaks im EPG ist ungefähr proportional zu der DNA-Menge, mit der die beteiligten Komponenten in der Mischung vertreten sind [5]. Anhand der Informationen zur Peakhöhe können die Spurenbeteiligten in eine Haupt- und eine Nebenkomponente unterschieden werden. Bei der Dekonvolution der 2-Personen-Mischspuren zeigte sich eine Abhängigkeit einer korrekten Ableitung vom Mischungsverhältnis der Komponenten. Dabei wurden die besten Ergebnisse bei den Mischspuren erzielt, die eine deutliche Unterscheidung von Haupt- und Nebenkomponente vorwiesen. In diesen wurde eine Übereinstimmung der von der Software abgeleiteten GTK mit dem tatsächlichen Genotyp häufiger beobachtet, was auf die Peakhöhendifferenz der in der Mischspur enthaltenen Allele zurückzuführen ist. Eine Berechnung der Dekonvolution von Spuren ohne deutliche Hauptkomponente führte zur Ableitung von GTK mit 2 der Personen nichtzugehörigen Allelen in einer höheren Anzahl von Systemen für beide Personen. Mischspuren, in denen die Komponenten anhand der Konvergenzanalyse von GPM4 anteilsähnlich vorliegen, stellen demnach eine Herausforderung für die Ableitung der GTK dar, da eine eindeutige Zuordenbarkeit der Allele anhand ihrer Peakhöhen durch die ähnlichen Fluoreszenzintensitäten nicht mehr zuverlässig erfolgen kann. In diesen Fällen ist eine Berechnung der Dekonvolution nicht sinnvoll.

Mit zunehmender Zahl an Spurenverursachenden konnte eine Abnahme der Effizienz hinsichtlich der korrekten Ableitung von Einzelprofilen festgestellt werden. Grundlegend beeinflussten folgende 


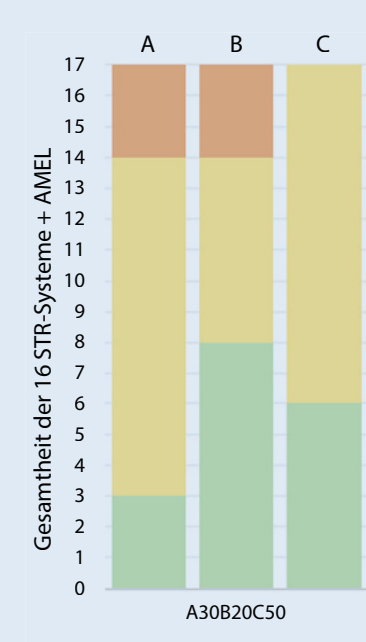

a
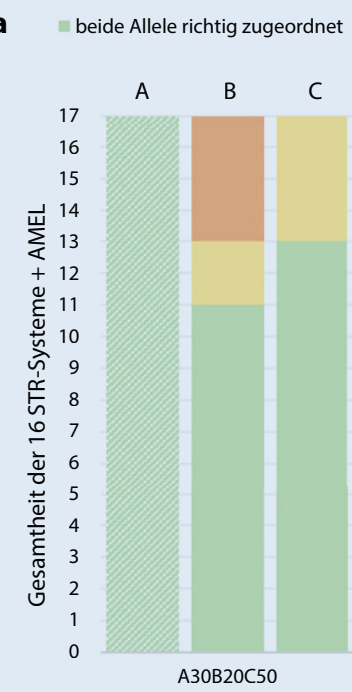

b

beide Allele richtig zugeordnet

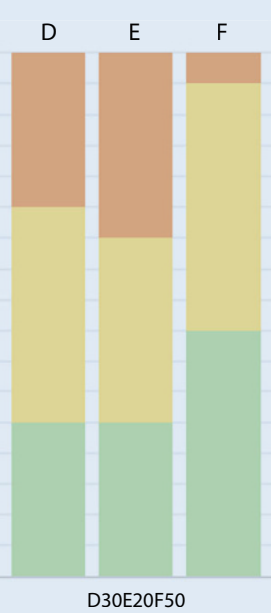

ein Allel falsch zugeordnet

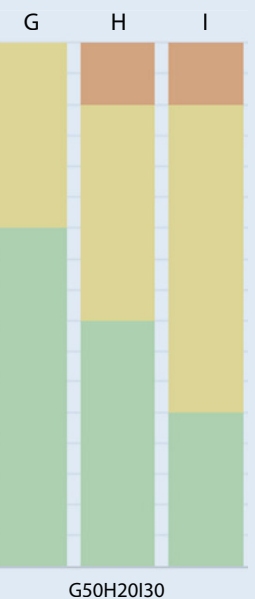

$\mathrm{G} 50 \mathrm{H} 20130$

\begin{abstract}
E F
\end{abstract}
= beide Allele falsch zugeordnet

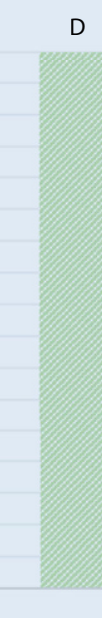

ein Allel falsch zugeordnet

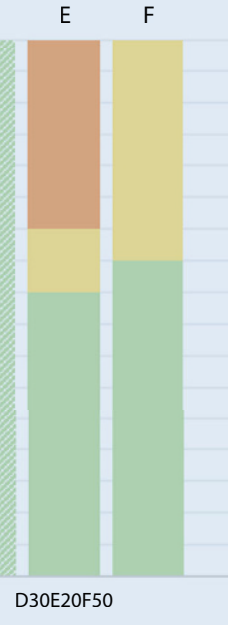

dnet

E beide Allele falsch zugeordnet

Abb. 4 ム Vergleich der Ableitungen der GTKin drei 3-Personen-Mischspuren bei 3 unbekannten Spurenlegenden ( $a$ ) und mit bekanntem Vergleichsmaterial (grünschraffiert, $b$ )

Faktoren die Ableitung der GTK beteiligter

Personen:

- Homo-/Heterozygotie im DNA-Profil der Spurenlegenden,

- Anteile der Nebenkomponenten,

- Qualität der Mischspuren [8, 12],

- Allele, die bei mehreren Personen im gleichen Marker auftreten.

Mit der Angabe des bekannten Profils der mittleren Komponenten konnten GTK abgeleitet werden, die in über $50 \%$ (teilweise bis zu $80 \%$ ) der untersuchten STRMarker mit dem tatsächlichen Personenprofil übereinstimmten. Durch das Setzen der mittleren Komponente konnte eine richtige Zuordnung der in der Mischspur detektierten Allele anhand von Peakhö- hen mit einer höheren Wahrscheinlichkeit erfolgen, da die Fluoreszenzintensitäten der Allelpeaks von Hauptkomponente und anteilig am niedrigsten vorliegender Komponente eine unterscheidbare Höhe aufweisen sollten.

\section{Fazit für die Praxis}

Die Dekonvolution eignet sich v. a. bei 2-oder 3-Personen-Mischspuren, in denen die DNAProfile der Spurenbeteiligten einen hohen Grad an Heterozygotie und einen geringen Grad an Allelüberlagerungen aufweisen, besonders gut. Liegt ein deutlicher DNA-Mengen-Unterschied von Haupt- und Nebenkomponente in einer 2-Personen-Mischspur vor, ist eine Berechnung mittels Genoproof ${ }^{\circledR}$ Mixture 4 von Vorteil. Des Weiteren bietet sich eine Dekonvolution von 3-Personen-Misch- spuren an, wenn für diese Spur Vergleichsmaterial einer beispielsweise berechtigten oder tatverdächtigen Person zur Verfügung steht. Die Dekonvolution einer Mischspur mit 3 unbekannten Spurenlegenden kann nur u.U. als eine Hilfestellung bei der Ermittlung des Einzelprofils für die Hauptkomponente dieser 3-Personen-Spur eingesetzt werden.

Die Dekonvolution mittels GPM4 ist in erster Linie abhängig von der Qualität der eingepflegten Daten und sollte als unterstützendes Tool nur in Spuren eingesetzt werden, in denen stochastische Effekte kaum eine Rolle spielen. Demnach empfiehlt es sich nicht, GPM4 bei der Untersuchung degradierten Spurenmaterials einzusetzen.

Folgende Kriterien helfen zu entscheiden, wann eine Berechnung der Dekonvolution sinnvoll ist, bzw. ob die abgeleiteten Genotypkonstellationen (GTK) als tatsächliche GTK anzunehmen sind:

- geringe Unterschiede in den Peakhöhenverhältnissen der Allele im Doppelansatz,

- die Anzahl der für die Komponente angenommenen Genotypen und deren jeweilige Wichtungen,

- der Abstand zwischen dem an 1. und 2. Position gewichteten Genotyp,

- die von GPM4 angenommenen Mischungsverhältnisse,

- eine über $>90 \%$ gewichtete GTK.

Die Ergebnisse der Berechnungen sollten dabei als hinweisgebend bzw. bestätigend zur subjektiv durchgeführten Merkmalsprofilableitung betrachtet werden.

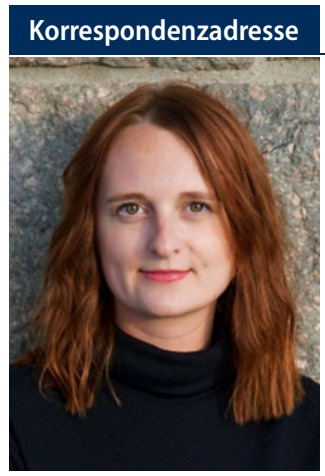

\section{Beate Dika}

Institut für Rechtsmedizin, Universität Leipzig Johannisallee 28, 04103 Leipzig, Deutschland beate.dika@medizin.uni-leipzig.de

Funding. Open Access funding enabled and organized by Projekt DEAL. 


\section{Einhaltung ethischer Richtlinien}

Interessenkonflikt. B. Dika, J. Dreßler, J. Edelmann und M. Kohl geben an, dass kein Interessenkonflikt besteht.

Alle beschriebenen Untersuchungen am Menschen oder an menschlichem Gewebe wurden mit Zustimmung der zuständigen Ethikkommission (Verfahrensnr. 45320-ek), im Einklang mit nationalem Recht sowie gemäß der Deklaration von Helsinki von 1975 (in der aktuellen, überarbeiteten Fassung) durchgeführt. Von allen Studienteilnehmenden liegt eine Einverständniserklärung vor.

Open Access. Dieser Artikel wird unter der Creative Commons Namensnennung 4.0 International Lizenz veröffentlicht, welche die Nutzung, Vervielfältigung, Bearbeitung, Verbreitung und Wiedergabe in jeglichem Medium und Format erlaubt, sofern Sie den/die ursprünglichen Autor(en) und die Quelle ordnungsgemäß nennen, einen Link zur Creative Commons Lizenz beifügen und angeben, ob Änderungen vorgenommen wurden.

Die in diesem Artikel enthaltenen Bilder und sonstiges Drittmaterial unterliegen ebenfalls der genannten Creative Commons Lizenz, sofern sich aus der Abbildungslegende nichts anderes ergibt. Sofern das betreffende Material nicht unter der genannten Creative Commons Lizenz steht und die betreffende Handlung nicht nach gesetzlichen Vorschriften erlaubt ist, ist für die oben aufgeführten Weiterverwendungen des Materials die Einwilligung des jeweiligen Rechteinhabers einzuholen.

Weitere Details zur Lizenz entnehmen Sie bitte der Lizenzinformation auf http://creativecommons.org/ licenses/by/4.0/deed.de.

\section{Literatur}

1. Butler JM (2014) Advanced Topics in Forensic DNA Typing: Interpretation, 1. Aufl. Elsevier academic Press, S 70-80. https://doi.org/10.1016/C2011-007649-4

2. Dror IE, Hampikian G (2011) Subjectivity and bias in forensic DNA mixture interpretation. Sci Justice 51:204-208

3. Gelman A, Rubin DB (1996) Markov chain Monte Carlo methods in biostatistics. Stat Methods Med Res 5:339-355

4. Götz FM, Schönborn H, Borsdorf V, Pflugbeil A-M, Labudde D (2017) GenoProof Mixture 3-New software and process to resolve complex DNA mixtures. Forensic Sci Int Genet Suppl Ser 6:e549-e551

5. Graversen, T., Lauritzen, S. (2013): Estimation of parameters in DNA mixture analysis. Journal of Applied Statistics 40.11:2423-243

6. Miller CR, Joyce P, Waits LP (2002) Assessing Allelic Dropout and Genotype Reliability Using Maximum Likelihood. Nat Genet 160:357-366

7. qualitype (2019): Benutzerdokumentation GenoProof ${ }^{(8)}$ Mixture 3 Version 3.1.2. qualitype GmbH Dresden. S. 33+34, S. 52+53, S. 185-200, S. 203-205

8. Schneider PM, Bender K, Mayr WR, Parson W, Hoste B, Decorte R, Cordonnier J, Vanek D, Morling N, Karjalainen M, Carlotti M-PC, SabatierM, HohoffC, Schmitter H, Pflug W, Wenzel R, Patzelt D,

\section{Deconvolution of DNA mixtures using a fully continuous model}

Background: When examining mixed traces stochastic effects can impair the assessment of a trace. Therefore, more and more software-based solutions are finding their way into trace investigation, which are intended to provide assistance in the derivation of individual profiles by taking biological parameters into account. In this study, a scientific validation of the mixed trace analysis software Genoproof ${ }^{\circledR}$ Mixture 4 (GPM4, Qualitype GmbH, Dresden, Deutschland) was performed.

Material and methods: The 2-person and 3-person mixtures present in different mixture ratios were artificially generated using isolated CD4 ${ }^{+}$lymphocytes from nine donors. After STR profiles were generated, the mixtures were evaluated for deconvolution using GPM4.

Results: In the 2-person mixtures with clear distinction of major and minor components, the correct genotype constellation (GTC) of the components was derived by the software in the vast majority of STR systems examined, often with a weighting of $>90 \%$. In the more proportionally similar mixed traces, inapplicable allele derivations were increasingly observed. A decrease in performance in terms of deriving the correct GTC was shown by the 3-person mixture traces. Factors such as mixture composition, homozygosity and heterozygosity in the genetic profiles were shown to influence the separation of the mixture traces.

Discussion: Mixed traces that do not allow clear differentiation of major and minor components present a difficulty in deconvolution. In these cases, it is difficult to differentiate the peak heights of detected alleles, because they have comparable intensity when the proportions of both components are equal. A clear DNA quantity difference in a 2-person mixed trace is advantageous for the calculation.

\section{Keywords}

Forensic molecular genetics · Analysis of DNA mixtures · Deconvolution · Probabilistic calculation model $\cdot$ Genoproof Mixture

Lessig R, Dobrowolski P, O'Donnell G, Garafano L, Dobosz M, De Knijff P, Mevag B, Pawlowski R, Gusmão L, VideCM, Alonso AA, García Fernández O, Sanz NP, Kihlgreen A, Bär W, Meier V, Teyssier A, Coquoz R, Brandt C, Germann U, Gill P, Hallett J, Greenhalgh M (2004) STR analysis of artificially degraded DNA-results of a collaborative European exercise. Forensic Sci Int 139:123-134

9. Schneider PM, Fimmers R, Keil W, Molsberger G, Patzelt D, Pflug W, Rothämel T, Schmitter $H$, Schneider H, Brinkmann B (2006) Allgemeine Empfehlungen der Spurenkommission zur Bewertung von DNA-Mischspuren. Rechtsmedizin 16:401-404

10. Tucker VC, Hopwood AJ, Sprecher CJ, McLaren RS, Rabbach DR, Ensenberger MG, Thompson JM, Storts DR (2011) Developmental validation of the PowerPlex ${ }^{\circledR}$ ESI 16 and PowerPlex ${ }^{\circledR}$ ESI 17 Systems: STR multiplexes for the new European standard. Forensic Sci Int Genet 5(5):436-448

11. Tucker VC, Hopwood AJ, Sprecher CJ, McLaren RS, Rabbach DR, Ensenberger MG, Thompson JM, Storts DR (2012) Developmental validation of the PowerPlex ${ }^{\circledR}$ ESX 16 and PowerPlex ${ }^{\circledR}$ ESX 17 Systems. Forensic Sci Int Genet 6(1):124-131

12. Ulbrich W, Anslinger $K$, Bäßler $G$, Eckert M, Fimmers R, Hohoff C, Kraft M, Leuker C, Molsberger G, Pich U, Razbin S, Schneider H, Templin M, Wächter A, Weirich V, Zierdt H, Schneider PM (2016) Gemeinsame Empfehlungen der Projektgruppe „Biostatistische DNA-Berechnungen“ und der Spurenkommission zur biostatistischen Bewertung von DNA-analytischen Befunden. Rechtsmedizin 26:291-298

13. Walsh PS, Fildes NJ, Reynolds R (1996) Sequence analysis and characterization of stutter products at the tetranucleotide repeat locus vWA. Nucl Acids Res 24(14):2807-2812 